\title{
Early Social Experience Is Critical for the Development of Cognitive Control and Dopamine Modulation of Prefrontal Cortex Function
}

\author{
Petra JJ Baarendse', Danielle S Counotte ${ }^{2}$, Patricio O'Donnell ${ }^{2}$ and Louk JMJ Vanderschuren ${ }^{* 1,3}$ \\ 'Department of Neuroscience and Pharmacology, Rudolf Magnus Institute of Neuroscience, UMC Utrecht, Utrecht, The Netherlands; \\ ${ }^{2}$ Department of Anatomy and Neurobiology, University of Maryland School of Medicine, Baltimore, MD, USA; ${ }^{3}$ Faculty of Veterinary Medicine, \\ Division of Behavioural Neuroscience, Department of Animals in Science and Society, Utrecht University, Utrecht, The Netherlands
}

\begin{abstract}
Social experiences during youth are thought to be critical for proper social and cognitive development. Conversely, social insults during development can cause long-lasting behavioral impairments and increase the vulnerability for psychopathology later in life. To investigate the importance of social experience during the juvenile and early adolescent stage for the development of cognitive control capacities, rats were socially isolated from postnatal day 21 to 42 followed by re-socialization until they reached adulthood. Subsequently, two behavioral dimensions of impulsivity (impulsive action in the five-choice serial reaction time task (5-CSRTT) and impulsive choice in the delayed reward task) and decision making (in the rat gambling task) were assessed. In a separate group of animals, long-lasting cellular and synaptic changes in adult medial prefrontal cortex (PFC) pyramidal neurons were determined following social isolation. Juvenile and early adolescent social isolation resulted in impairments in impulsive action and decision making under novel or challenging circumstances. Moreover, socially isolated rats had a reduced response to enhancement of dopaminergic neurotransmission (using amphetamine or GBR I 2909) in the 5-CSRTT under challenging conditions. Impulsive choice was not affected by social isolation. These behavioral deficits were accompanied by a loss of sensitivity to dopamine of pyramidal neurons in the medial PFC. Our data show long-lasting deleterious effects of early social isolation on cognitive control and its neural substrates. Alterations in prefrontal cognitive control mechanisms may contribute to the enhanced risk for psychiatric disorders induced by aberrations in the early social environment.

Neuropsychopharmacology (2013) 38, |485-|494; doi:I0.1038/npp.2013.47; published online I3 March 2013
\end{abstract}

Keywords: social isolation; adolescence; prefrontal cortex; impulsivity; decision making; dopamine

\section{INTRODUCTION}

Humans and many other mammals are social species. Marked changes in social behavior take place during postweaning development (ie, childhood and adolescence in humans, equivalent to the juvenile and adolescent stages in rodents), including increased complexity of the social repertoire and a profound increase in peer-directed social interactions, signified by an abundance of social play behavior (Blakemore, 2008; Nelson et al, 2005; Spear, 2000; Vanderschuren et al, 1997). Social play behavior is thought to facilitate neural and behavioral development to equip the individual with a flexible and adaptive behavioral repertoire (Pellis and Pellis, 2009; Špinka et al, 2001). Conversely, disruptions in the early social environment can result in persistent neurobiological changes that may

*Correspondence: Professor LJMJ Vanderschuren, Faculty of Veterinary Medicine, Division of Behavioural Neuroscience, Department of Animals in Science and Society, Utrecht University, Yalelaan 2, Utrecht 3584 CM, The Netherlands. Tel: +3I 30 2535239, Fax: +3I 30 2537997, E-mail: I.j.m.j.vanderschuren@uu.nl

Received 26 October 2012; revised 18 January 2013; accepted 4 February 2013; accepted article preview online 12 February 2013 increase the vulnerability for psychiatric diseases later in life (Cacioppo and Hawkley, 2009; Paus et al, 2008).

The juvenile and adolescent stages are critical periods of neural maturation (Casey et al, 2005; Counotte et al, 2010; Paus et al, 2008). In particular, the prefrontal cortex (PFC) continues to develop until early adulthood in humans (Casey et al, 2005; Paus et al, 2008) and rodents (Counotte et al, 2010; Tseng and O'Donnell, 2005, 2007). As adverse events are likely to maximally impact on brain regions that are in transition (Andersen, 2003), early environmental insults, such as social isolation, may profoundly affect PFC function (Leussis et al, 2008; Makinodan et al, 2012). Indeed, isolation rearing studies, in which animals are socially isolated after weaning, have consistently shown changes in PFC function, including disrupted synaptic plasticity, as well as decreases and increases in PFC dopamine and serotonin signaling, respectively (for review see Fone and Porkess, 2008). However, isolation rearing comprises continuous social isolation from weaning onwards, so that the specific period of social isolation that is critical for inducing these neurobehavioral changes is largely unknown. As the PFC is essential for cognitive control functions, such as impulse control and decision 
making (Miller and Cohen, 2001; Robbins and Arnsten, 2009), we hypothesized that social play behavior facilitates the development of these functions. To test this hypothesis, we assessed the long-term cognitive effects of social isolation during postnatal day (PND) 21-42. This age range is comparable to childhood and early adolescence in humans (McCutcheon and Marinelli, 2009; Spear, 2000), and it is the period when social play behavior peaks in rats (Panksepp, 1981). Impulsive action was studied using the five-choice serial reaction time task (5-CSRTT; Robbins, 2002), and the delayed reward task (DRT) was used to measure impulsive choice (Evenden and Ryan, 1996). Decision making was measured using the rat gambling task (rGT; Zeeb et al, 2009), a rodent analog of the human Iowa gambling task (Bechara et al, 1994). To characterize the role of monoamine neurotransmission in the long-term behavioral effects of early adolescent social isolation, we investigated the effects of amphetamine, as well as selective inhibitors of the reuptake of dopamine, serotonin, and noradrenaline on impulsivity and decision making. Moreover, we used slice electrophysiology to determine longlasting cellular and synaptic changes in medial PFC (mPFC) pyramidal neurons in adult animals following social isolation.

\section{MATERIALS AND METHODS}

\section{Animals}

Male Lister Hooded (behavioral experiments; Harlan, The Netherlands) or Long-Evans rats (electrophysiology experiments; Charles Rivers Laboratories, USA) arrived at 21 days of age and were housed either socially (SOC) or individually (ISO) the day after arrival. Rats were housed under reversed lighting conditions (lights on at 1900 hours) for the behavioral experiments and normal light/dark conditions (lights on at 0700 hours) for the electrophysiology experiments. Rats of the ISO group were re-socialized, ie, housed together with an animal of the same treatment group, on day 43. Behavioral testing and electrophysiological recordings started at 12 weeks of age. A separate cohort of animals was used for every experiment (5-CSRTT, DRT, rGT, and mPFC recordings). During behavioral training and testing, rats were placed on a restricted diet of $14 \mathrm{~g}$ chow per day. All experiments were approved by the Animal Ethics Committee of the Utrecht University and the University of Maryland School of Medicine Institutional Animal Care and Use Committee and were conducted in agreement with Dutch laws (Wet op de Dierproeven, 1996), European regulations (Guideline 86/609/EEC), and the United States Public Health Service Guide for the Care and Use of Laboratory Animals.

\section{Apparatus}

Behavioral testing (5-CSRTT, DRT, and rGT) was conducted in operant conditioning chambers (Med Associates, USA) enclosed in sound-insulating, ventilated boxes. Set in the curved wall of each box was an array of five nose poke holes. Each nose poke unit was equipped with an infrared detector and a yellow stimulus light. Food pellets (45 mg, Formula P; Bio-Serv) could be delivered at the opposite wall via a dispenser. The chamber could be illuminated by a white house light, mounted in the center of the roof. Online control of the apparatus and data collection was performed using MEDPC (Med Associates).

\section{Five-Choice Serial Reaction Time Task}

Rats were trained to respond to a brief visual stimulus presented randomly in one of the five nose poke units to obtain a food reward (Baarendse and Vanderschuren, 2012; Robbins, 2002; Van Gaalen et al, 2006a). Each session consisted of 100 trials or $30 \mathrm{~min}$, whichever occurred first. A trial started with an intertrial interval (ITI) of $5 \mathrm{~s}$, followed by $1 \mathrm{~s}$ illumination of one of the five apertures and a $2 \mathrm{~s}$ limited hold. A nose poke in the illuminated aperture (correct response) was rewarded with one food pellet. Nose pokes made during the ITI (premature responses) were recorded as a measure of impulsivity. An incorrect response, failure to respond (error of omission) and premature response, resulted in no food delivery and a time-out period with the house light illuminated for $5 \mathrm{~s}$. After training to baseline performance, rats were exposed to various task manipulations, such as increasing the ITI to $7 \mathrm{~s}$ (long ITI), decreasing the stimulus duration to $0.5 \mathrm{~s}$ (short stimulus duration), and increasing the performance load by decreasing the ITI to $2.5 \mathrm{~s}$ (high event rate). The following measures were recorded: (1) premature responses (number of responses into one of the holes during the ITI); (2) accuracy (the percentage of correct responses [(number correct responses $) /($ correct + incorrect responses $) \times 100])$; and (3) omission errors (the total number of omitted trials during a session).

\section{Delayed Reward Task}

Each session was divided into five blocks of 12 trials. Nose poking into one illuminated nose poke hole resulted in the immediate delivery of a small reward (one food pellet), whereas nose poking into one other illuminated hole resulted in the delivery of a large, delayed reward (four food pellets) (Baarendse and Vanderschuren, 2012; Evenden and Ryan, 1996; Van Gaalen et al, 2006b). The delays to the large reward increased within a session from 0 to $10,20,40$, and $60 \mathrm{~s}$ per block. After delivery of the reward or the choice phase time elapsed, an ITI commenced. The holes associated with the small and large reward were counterbalanced between animals. Following baseline performance, rats were exposed to a task manipulation that consisted of increased delays, ie, $0,20,40,60$, and $80 \mathrm{~s}$ per block. The percentage choice for the large reward as a function of delay was calculated as the [(number of choices for the large reinforcer)/(number choices large + small reinforcers) $\times 100]$.

\section{Rat Gambling Task}

In this task, rats are confronted with four choices differing in the probability and magnitude of reward (food) and punishment (time-out; Baarendse et al, 2013; Zeeb et al, 2009). Rats were tested once daily in a 30 -min session. A trial started with a 5-s ITI followed by illumination of holes $1,2,4$, and 5 for $10 \mathrm{~s}$. A response in an illuminated hole turned off all stimulus lights, and led to either the delivery 
of reward or the start of a punishment time-out. Animals were first tested in 10 free choice sample sessions, in which the first two choices for each option were rewarded, after which the reward and punishment contingencies associated with the four response options were introduced. The free sample sessions were followed by five forced-choice sessions before moving on to the full free choice task. Premature responses were punished by a 5-s time-out period, signaled by illumination of the house light. The reinforcement schedules were designed such that the optimal strategy, in terms of maximal number of food pellets earned per session, was to select the two-pellet option (P2), associated with a 10-s time-out period that occurs $20 \%$ of the time ( $80 \%$ chance of reward). The next best option is P1 ( $5 \mathrm{~s}$ time-out, $90 \%$ chance of reward). The two disadvantageous options were both associated with larger immediate gain (three or four pellets), but also longer time-out periods (P3: $30 \mathrm{~s}$ time-out, $50 \%$ chance of reward; P4: $40 \mathrm{~s}$ time-out; $40 \%$ chance of reward). The spatial locations of the pellet choice options were counterbalanced across subjects. Choice scores were calculated as the sum of the two advantageous options (average percentage choice of $\mathrm{P} 1$ and $\mathrm{P} 2$ ) and the sum of the two disadvantageous options (average percentage choice of P3 and P4).

\section{Drugs}

(+)-Amphetamine sulphate $(0.5 \mathrm{mg} / \mathrm{kg}$; O.P.G., The Netherlands), GBR 12909 dihydrochloride $(10 \mathrm{mg} / \mathrm{kg})$, atomoxetine hydrochloride $(3 \mathrm{mg} / \mathrm{kg})$, and citalopram hydrobromide ( $1 \mathrm{mg} / \mathrm{kg}$; Tocris Bioscience, UK) were dissolved in $0.9 \%$ saline (amphetamine, atomoxetine, and citalopram) or sterile water (GBR12909). Animals received five daily test sessions per week until stable patterns of performance were observed. Next, the effects of the task manipulations were assessed (5-CSRTT, DRT). Subsequently, the effects of the four drugs on performance in the 5-CSRTT, DRT, and rGT were tested. Drug tests were conducted on Tuesdays and Fridays with baseline training sessions on the other weekdays. Before the first drug test, all animals had been habituated twice to i.p. saline injections. Every animal in a cohort received a challenge with every drug, according to a Latin square design. Injections were given i.p., $30 \mathrm{~min}$ (5-CSRTT, rGT) or $10 \mathrm{~min}$ (DRT) before behavioral testing. We have previously demonstrated effects on impulsivity of these drugs using these doses and pretreatment intervals (Baarendse and Vanderschuren, 2012).

\section{Whole-Cell Recordings}

At 12 weeks of age, rats were anesthetized with chloral hydrate $(400 \mathrm{mg} / \mathrm{kg}$, i.p.) $15 \mathrm{~min}$ before decapitation. Brains were quickly removed and placed into ice-cold artificial CSF oxygenated with $95 \% \quad \mathrm{O}_{2}-5 \% \quad \mathrm{CO}_{2}$ and containing the following (in mM): $125 \mathrm{NaCl}, 25 \mathrm{NaHCO}_{3}, 10$ glucose, 3.5 $\mathrm{KCl}, 1.25 \mathrm{NaH}_{2} \mathrm{PO}_{4}, 0.5 \mathrm{CaCl}_{2}$, and $3 \mathrm{MgCl}_{2}$, pH 7.45 (295$300 \mathrm{mOsm})$. Coronal slices $(300 \mu \mathrm{m})$ containing the PFC were obtained with a vibratome in ice-cold aCSF and incubated in warm $\left(\sim 35^{\circ} \mathrm{C}\right)$ aCSF solution constantly oxygenated with $95 \% \mathrm{O}_{2}-5 \% \mathrm{CO}_{2}$ for at least $45 \mathrm{~min}$ before recording. Whole-cell recordings from layer $\mathrm{V}$ pyramidal neurons were made using standard electrophysiological techniques (Tseng et al, 2008). Patch electrodes (7-10 M $\Omega$ ) were filled with a solution containing $0.125 \%$ neurobiotin and the following (in $\mathrm{mM}$ ): $115 \mathrm{~K}$-gluconate, 10 HEPES, 2 $\mathrm{MgCl}_{2}, 20 \mathrm{KCl}, 2 \mathrm{MgATP}, 2 \mathrm{Na}-\mathrm{ATP}$, and $0.3 \mathrm{GTP}, \mathrm{pH}$ 7.25-7.30 (280-285 mOsm). All experiments were conducted at $33-35^{\circ} \mathrm{C}$.

Synaptic responses were tested in pyramidal neurons with electrical stimulation of layers I-II with a bipolar electrode made from a pair of twisted Teflon-coated tungsten wires (tips separated by $\sim 200 \mu \mathrm{m}$ ) and placed $\sim 500 \mu \mathrm{m}$ lateral to the vertical axis of the recorded neuron. Stimulation pulses $(0.02-0.4 \mathrm{~mA} ; 0.5 \mathrm{~ms})$ were delivered every $15 \mathrm{~s}$. Throughout the experiment, input resistance was measured with a single hyperpolarizing step and the cell was discarded when the input resistance changed more than $20 \%$ during the course of the experiment. The amplitude of evoked postsynaptic potentials (EPSPs) was measured with Clampfit 9.0 and averaged over 10 sweeps before and after $10 \mathrm{~min}$ of application of a mixture of SKF38393 and quinpirole. At the end of each experiment, the slices were placed in $4 \%$ paraformaldehyde and processed for DAB staining using standard histochemical techniques to verify morphology and location of the neurons.

\section{Data Analysis}

In the behavioral experiments, the initial group size was $n=12$. However, due to experimental errors and computer failure, the data of some drug challenges were excluded from the analysis. The final group size in the behavioral experiments was therefore $n=10-12$. An arcsine transformation was performed before analysis of the percentage of choice in the DRT and rGT to limit the effect of an artificially imposed ceiling. In the DRT, animals that did not show delay-dependent choice behavior $(0 \%$ choice for large reward at $0 \mathrm{~s}$ delay, $100 \%$ choice for large reward at 40 and $60 \mathrm{~s}$ delay) were excluded from the experiment. Data were analyzed by one- (5-CSRTT, EPSP amplitude) or two-factor (DRT, rGT) repeated-measures ANOVAs with task challenge (5-CSRTT), dopamine (EPSP amplitude), or drug treatment (5-CSRTT, DRT, rGT) and delay to large reinforcer (DRT) or choice (rGT) as within-subjects variables, and rearing condition (SOC-ISO) as betweensubjects variables. In case of statistically significant main effects, post-hoc comparisons were conducted using Paired samples or Student's $t$-tests. The level of probability for statistically significant effects was set at 0.05 .

\section{RESULTS}

\section{Impulsive Action (5-CSRTT): Baseline, Behavioral, and Pharmacological Challenges}

ISO and SOC rats acquired the 5-CSRTT at comparable rates (sessions needed to reach baseline performance: SOC $36.3 \pm 2.0$, ISO $33.3 \pm 1.7 ; \mathrm{F}[$ group $](1,22)=0.47, p=0.26$ ), and there were no differences in behavior under baseline conditions between ISO and SOC rats (Figure $1 \mathrm{a}-\mathrm{c}$ ). Exposure to task challenges in the 5-CSRTT significantly affected premature responses, ie, impulsive action (Figure 1a; task challenge: $\mathrm{F}(3,66)=107.51, p<0.001$ ), 

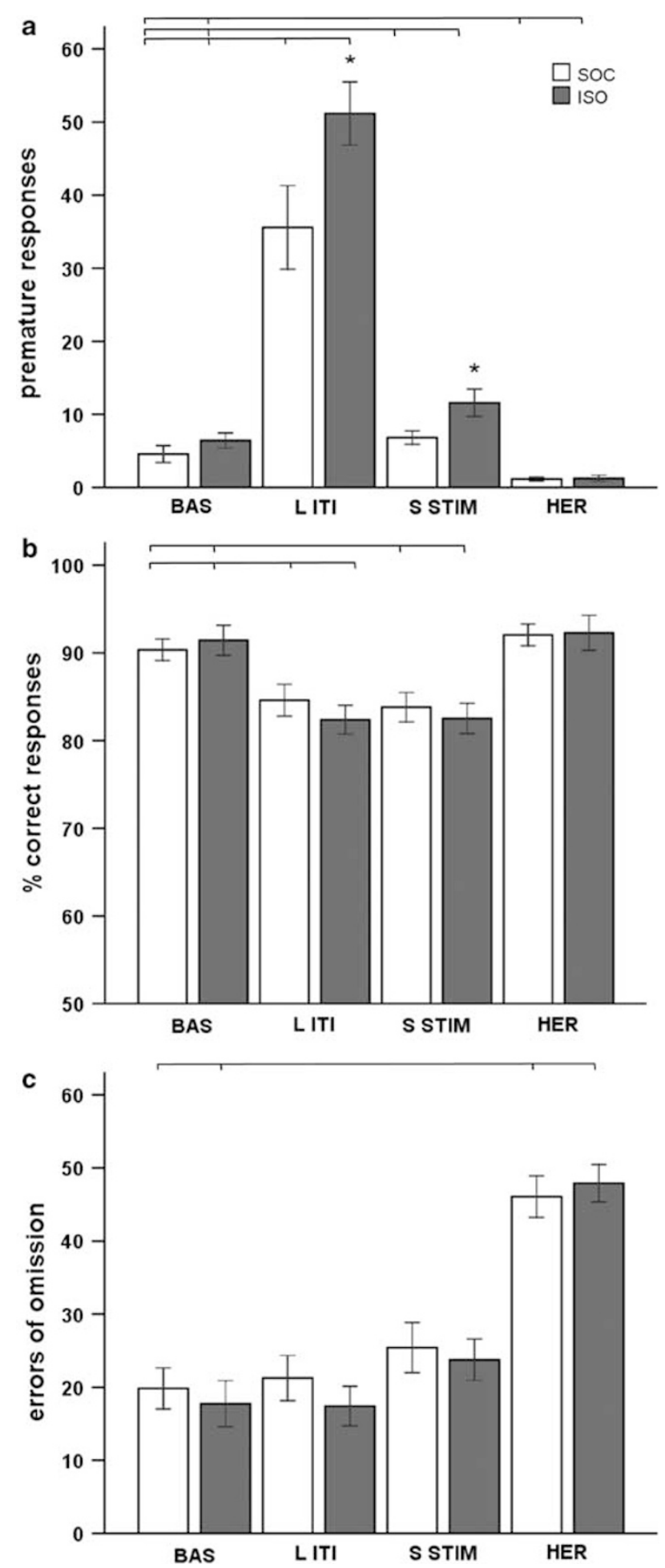

Figure I Effect of social isolation during postnatal day 21-42 followed by re-socialization on adult performance in the 5-choice serial reaction time task. (a) Amount of premature responses, ie, impulsive action, (b) percentage of correct responses, ie, accuracy and (c) errors of omission, under baseline conditions (BAS: visual stimulus presented $5 \mathrm{~s}$ after trial initiation, I s stimulus duration) or long intertrial interval ( $L$ ITI: visual stimulus presented $7 \mathrm{~s}$ after trial initiation), short stimulus duration (S STIM: $0.5 \mathrm{~s}$ stimulus duration), high event rate (HER: intertrial interval $2.5 \mathrm{~s}$ ). Group size was $n=12$. SOC = socially housed rats during PND $21-42$, $\mathrm{ISO}=$ socially isolated rats during PND $2 \mathrm{I}-42$. Asterisk indicates $p<0.05$ compared with SOC. Line on top of bar indicates $p<0.05$ compared with baseline. All data are expressed as mean \pm SEM.

percentage of correct responses (Figure 1b; task challenge: $\mathrm{F}(3,66)=27.41, p<0.001)$, and errors of omission (Figure 1c; task challenge: $\mathrm{F}(3,66)=72.95, p<0.001$ ) in both groups. However, when the ITI was increased to $7 \mathrm{~s}$ or the stimulus duration decreased to $0.5 \mathrm{~s}$, ISO rats made more premature responses (challenge $\times$ isolation: $F(3,66)=3.48$, $p<0.03$; Figure 1a). These task challenges did not differentially affect task accuracy (Figure 1b) or errors of omission (Figure 1c) in ISO vs SOC rats (challenge $\times$ isolation: F[\% correct responses] $(3,66)=0.66$, NS; F[errors of omission] $(3,66)=0.58$, NS). No difference between groups was observed when rats were exposed to a high event rate (decrease of ITI to $2.5 \mathrm{~s} ; p>0.05$ ).

Pharmacological challenges under baseline conditions significantly affected premature responses (Figure 2a; drug: $\mathrm{F}(4,80)=18.05, p<0.001)$, percentage of correct responses (Figure 2b; drug: $\mathrm{F}(4,88)=17.44, p<0.001$ ), and errors of omission (Figure $2 \mathrm{c}$; drug: $\mathrm{F}(4,88)=34.3, p<0.001$ ), without an effect of early adolescent social isolation (drug $\times$ isolation: $\mathrm{F}$ [premature responses] $(4,80)=1.14, \mathrm{NS} ; \mathrm{F}[\%$ correct responses] $(4,88)=2.14$, NS; F[errors of omission] $(4,88)=0.22$, NS). Under baseline conditions, amphetamine and the selective dopamine reuptake inhibitor GBR12909 increased premature responding, and the selective noradrenaline reuptake inhibitor atomoxetine decreased the amount of premature responses in both groups $(p<0.05)$. The percentage of correct responses was decreased by amphetamine and GBR12909, whereas atomoxetine increased errors of omission $(p<0.05)$. Although no overall effect of social isolation was observed (drug $\times$ isolation: $\mathrm{F}[$ premature responses $](4,80)=1.14$, NS; $\mathrm{F}[\%$ correct responses $](4,88)=2.14$, NS; $\mathrm{F}[$ errors of omission $](4,88)$ $=0.22$, NS), the selective serotonin reuptake inhibitor citalopram decreased premature responding in ISO rats, and decreased accuracy in the SOC group (drug: $\mathrm{F}$ [premature responses] $(4,80)=18.05, p<0.001 ; \mathrm{F}[\%$ correct responses $](4,88)=17.44, p<0.001$; post hoc $p<0.05)$.

Under a long ITI, amphetamine and GBR12909 increased premature responding (Figure 2d; drug: $\mathrm{F}(4,88)=26.67$, $p<0.001)$, but this effect was found in the SOC group only (drug $\times$ isolation: $\mathrm{F}(4,88)=2.42, p<0.05$ ), as no enhancement of premature responding by amphetamine and GBR12909 was observed in ISO rats $(p>0.05)$. Citalopram and atomoxetine decreased premature responding in both groups (both post-hoc $p<0.001$; Figure 2d). Atomoxetine increased errors of omission, whereas both citalopram and atomoxetine increased the percentage of correct responses under the long ITI (drug: F[\% correct responses] $(4,88)=31.99, p<0.001 ; \mathrm{F}$ [errors of omission $](4,88)=9.92$, $p<0.001$ ), but not differentially between groups (drug $\times$ isolation $\mathrm{F}[\%$ correct responses] $(4,88)=2.1$, NS; F[errors of omission $](4,88)=0.21$, NS; Figure $2 \mathrm{e}$ and $\mathrm{f}$ ).

\section{Impulsive Choice (DRT): Baseline, Increased Delay, and Pharmacological Challenges}

Overall, both groups showed a delay-dependent decline in their choice for the large, delayed reward under baseline conditions (delay: $\mathrm{F}(4,80)=69.02, p<0.001$ ) without an effect of early adolescent social isolation (delay $\times$ isolation: $\mathrm{F}(4,80)=1.70$, NS; Figure 3a). As the behavioral deficits in the ISO animals in the 5-CSRTT were especially apparent 

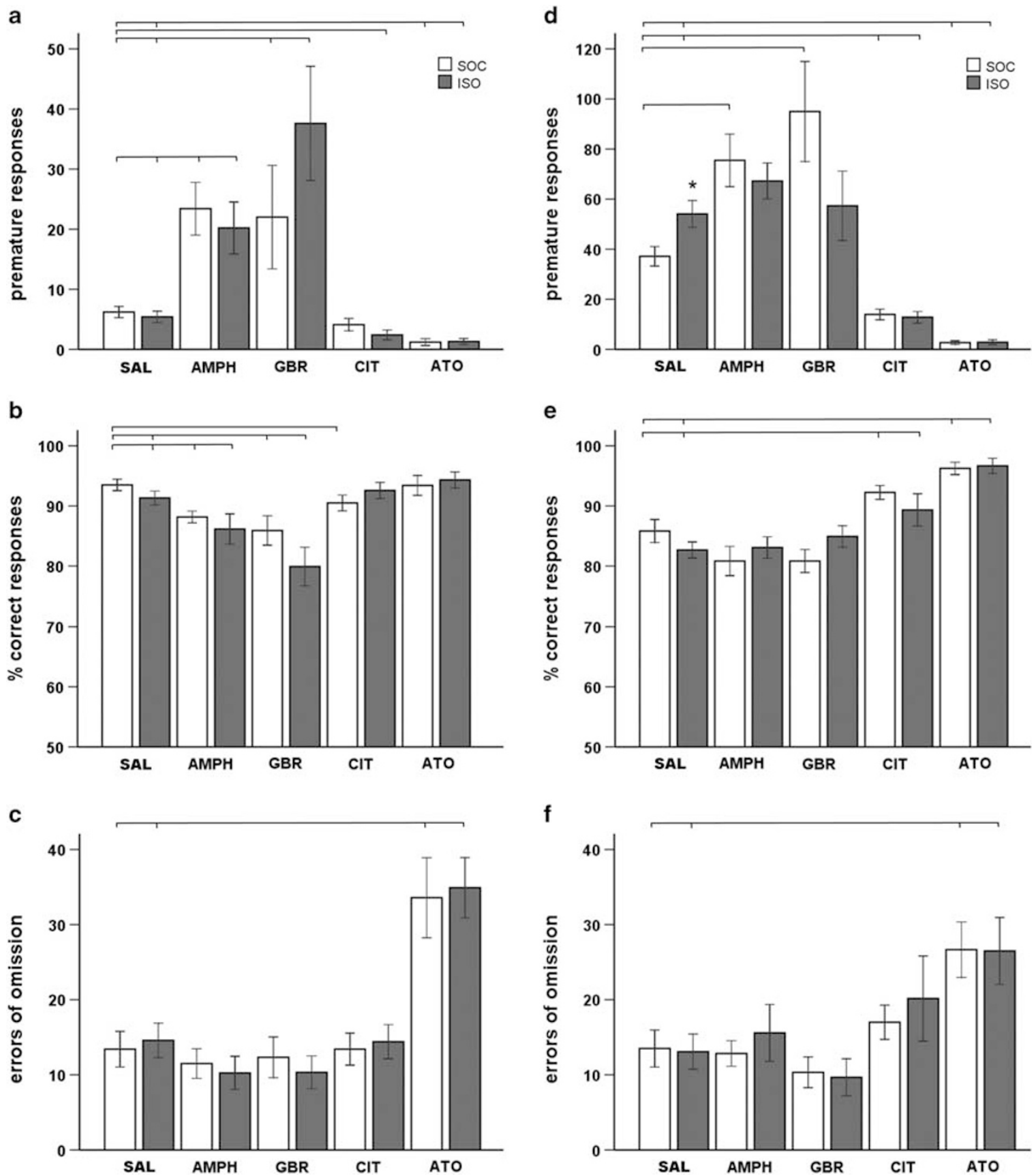

Figure 2 Effect of social isolation during postnatal day $21-42$ followed by re-socialization on adult performance in the 5-choice serial reaction time task. $(a, d)$ Amount of premature responses, ie, impulsive action; (b,e) percentage of correct responses, ie, accuracy; (c,f) errors of omission, under (a-c) baseline conditions (visual stimulus presented $5 \mathrm{~s}$ after trial initiation) or ( $\mathrm{d}-\mathrm{f}$ ) long ITI conditions (visual stimulus presented $7 \mathrm{~s}$ after trial initiation). Pharmacological challenges consisted of treatment with $0.5 \mathrm{mg} / \mathrm{kg}$ of amphetamine (AMPH), $10 \mathrm{mg} / \mathrm{kg}$ of the selective dopamine reuptake inhibitor GBRI2909 (GBR), I mg/ $\mathrm{kg}$ of the selective serotonin reuptake inhibitor citalopram (CIT), and $3 \mathrm{mg} / \mathrm{kg}$ of the selective noradrenaline reuptake inhibitor atomoxetine (ATO). In total, $n=10-12$ animals per treatment group were included in the analysis. SOC = socially housed rats during PND $21-42, I S O=$ socially isolated rats during PND $21-42$, SAL = saline treatment. Asterisk indicates $p<0.05$ compared with SOC group. Line on top of bar indicates $p<0.05$ compared with baseline or saline treatment. All data are expressed as mean \pm SEM.

when the animals were unexpectedly challenged with more demanding task conditions, we also assessed their behavior in the DRT when the delays were unexpectedly increased. Under these circumstances, there was no difference between ISO and SOC animals (delay: $\mathrm{F}(4,84)=42.29$, $p<0.001$; delay $\times$ isolation: $\mathrm{F}(4,84)=0.77$, NS; Figure $3 \mathrm{~b}$ ). Early social isolation did not affect the effects of amphetamine, GBR12909, citalopram, and atomoxetine on impulsive choice in the DRT (delay $x$ drug $\times$ isolation: $\mathrm{F}(4,84)=0.61$, NS). The effects of amphetamine, which enhanced the preference for the large reward (drug: $\mathrm{F}(1,21)=22.03, p<0.001$; delay $\times$ drug: $\mathrm{F}(4,84)=3.56$, $p<0.02)$, are shown in Figure 3c. Consistent with previous findings (Baarendse and Vanderschuren, 2012), GBR12909 

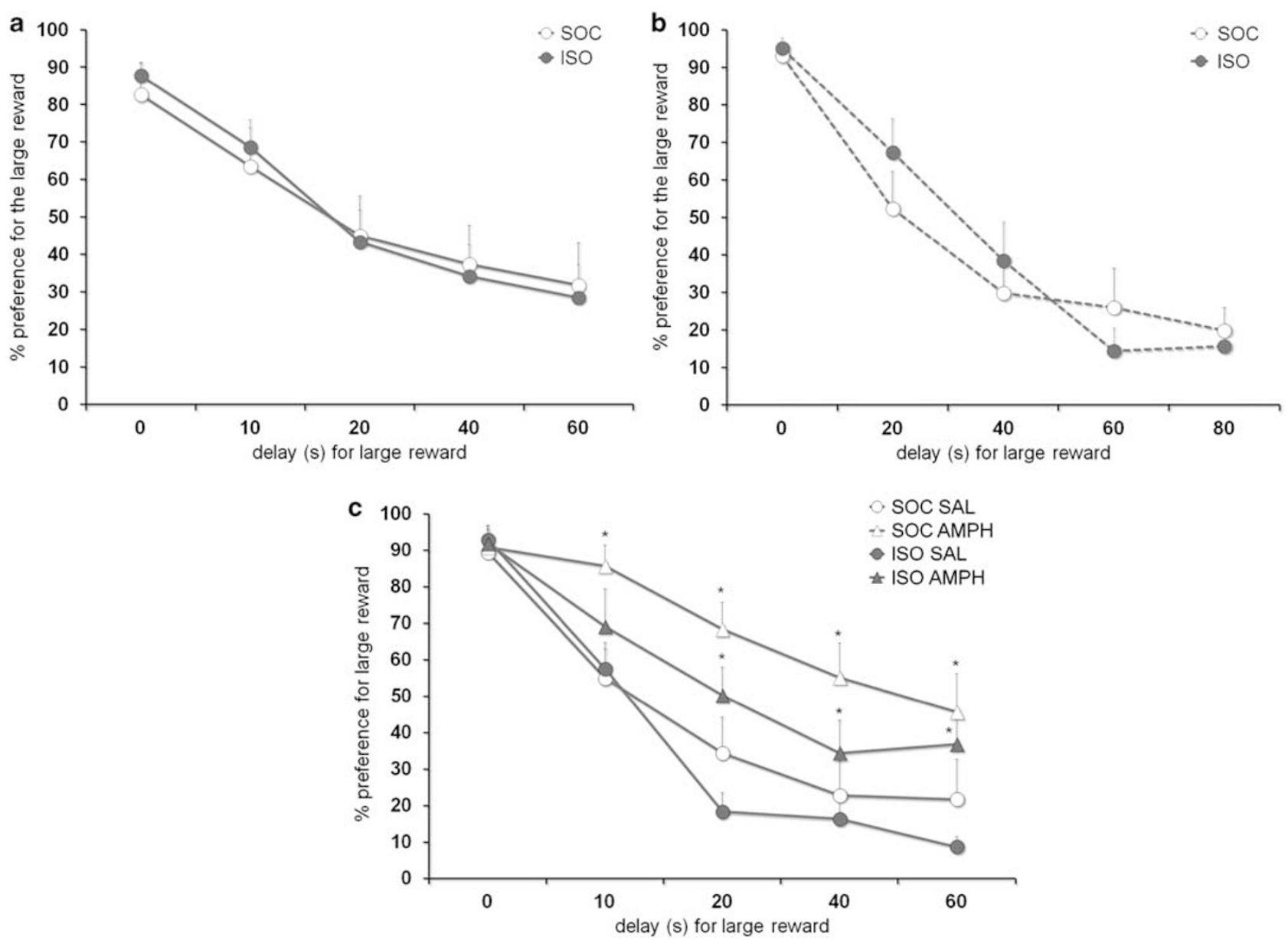

Figure 3 Effect of social isolation during postnatal day 2I-42 followed by re-socialization on impulsive choice in the DRT during adulthood. Percentage of choice for the large reward expressed per block of trials with increasing delays under (a) baseline, (b) increased delays, and (c) I mg/kg amphetamine challenge. In total, $n=10-12$ animals per treatment group were included in the analysis. AMPH =amphetamine, SOC $=$ socially housed rats during PND $2 \mathrm{I}-42$, ISO = socially isolated rats during PND $2 \mathrm{I}-42$. Asterisk indicates $p<0.05$ compared with saline treatment. All data are expressed as mean \pm SEM.

increased the percentage of choice for the large reward, ie, improved impulsive choice, whereas atomoxetine and citalopram did not influence choice behavior in the DRT (drug: GBR12909: $\mathrm{F}(1,19)=8.34, p<0.01$; atomoxetine: $\mathrm{F}(1,21)=0.08$, NS; citalopram: $\mathrm{F}(1,21)=3.66$, NS; data not shown).

\section{Decision Making (rGT): Acquisition, Baseline, and Pharmacological Challenges}

A detailed analysis of choice behavior per quartile of trials during the first free sampling choice session is depicted in Figure $4 \mathrm{a}$ and $\mathrm{b}$. SOC rats started with an equal preference for the advantageous $v s$ disadvantageous options during the first quartile of trials (Q1), and developed a preference for the advantageous options within the next quartiles of trials (Figure 4a; quartile $\times$ choice: $\mathrm{F}(3,33)=5.22, p<0.01$, posthoc Q1: NS, Q2-4: $p<0.02)$. Remarkably, ISO rats did not show this acquisition curve in decision making in the first rGT session during adulthood (Figure 4b; quartile $\times$ choice: $F(3,33)=0.28, \mathrm{NS})$. This effect of social isolation was supported by a significant quartile by group interaction (quartile $\times$ group: $F(3,66)=2.92, p<0.05)$. Analysis of choice behavior during the 10 free sampling choice sessions revealed that both groups acquired the task at comparable rates (F[group] $(1,21)=0.89$, NS; F[group $\times$ session] $(9,189)=1.85, p=0.06)$, with no between-group differences in performance apparent from session 3 onwards (data not shown).

Neither amphetamine nor one of the monoamine reuptake inhibitors, ie, GBR12909, citalopram, atomoxetine, altered the choice for the advantageous $v s$ disadvantageous options in the social or isolated group (drug: $\mathrm{F}$ [advantageous $](4,36)=0.73, p=\mathrm{NS} ; \mathrm{F}$ [disadvantageous $]$ $(4,40)=0.86, p=\mathrm{NS}$; Figure $4 \mathrm{c})$. Moreover, there was no difference in the effect of pharmacological manipulations between both groups (drug $\times$ group, $\mathrm{F}(4,76)=0.51, \mathrm{NS}$ ) . Analysis of choice behavior for the separate pellet options showed that amphetamine increased choice for P1 and decreased choice for P2 (drug: $\mathrm{F}(1,60)=6.22, p=0.02$ ), but this effect was not different between both groups. GBR12909, atomoxetine, and citalopram did not alter choice behavior for the four pellet options. This pattern of effects is consistent with our previous findings on the effects of amphetamine and monoamine reuptake inhibitors on behavior in the rGT (Baarendse et al, 2013). 

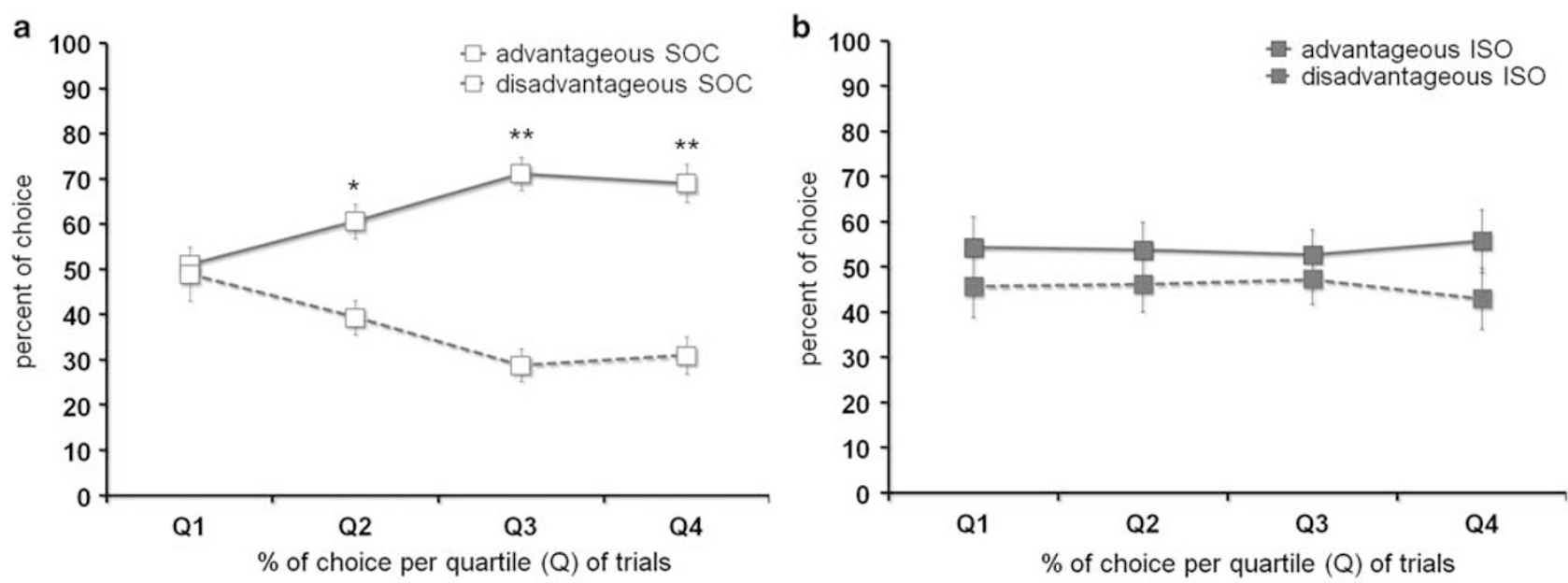

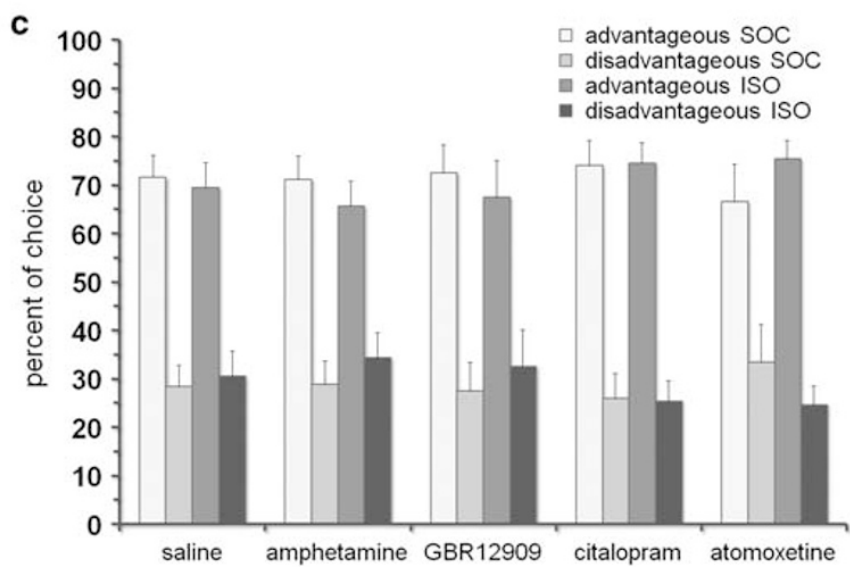

Figure 4 Effect of social isolation during postnatal day 21-42 followed by re-socialization on decision making in the rat gambling task during adulthood. Percentage of choice for the advantageous vs disadvantageous options during $(a, b)$ the first free sample choice session, and (c) pharmacological challenges after stable baseline performance. Pharmacological challenges consisted of I mg/kg of amphetamine, $10 \mathrm{mg} / \mathrm{kg}$ GBR $12909,3 \mathrm{mg} / \mathrm{kg}$ citalopram, and $3 \mathrm{mg} / \mathrm{kg}$ atomoxetine. In total, $n=10-12$ animals per treatment group were included in the analysis. SOC = socially housed rats during PND $21-42$, ISO = socially isolated rats during PND $21-42$. Asterisk indicates $p<0.05$ and double asterisks indicate $p<0.01$ difference between options. All data are expressed as mean \pm SEM.

\section{Whole-Cell Patch-Clamp Recordings}

Functional differences following social isolation in early adolescence was studied using whole-cell recordings in layer $\mathrm{V}$ pyramidal cells in mPFC (Figure 5). Early adolescent social isolation did not change any of the basic membrane properties (resting membrane potential, rheobase (current to fire an action potential), and input resistance) of $\mathrm{mPFC}$ layer $\mathrm{V}$ pyramidal cells (data not shown). In SOC animals, stimulation of dopamine receptors with bath application of the D1 agonist SKF38393 $(5 \mu \mathrm{M})$ and the D2 agonist quinpirole $(1 \mu \mathrm{M})$ decreased the amplitude of synaptic responses, whereas this effect was absent in ISO rats (Figure 5; dopamine $\times$ group, $\mathrm{F}(1,29)=9.49, p=0.004$; dopamine, $\mathrm{F}(1,29)=0.21$, NS). Locations of the recording sites are shown in Figure 5f. Most recorded cells were in infralimbic cortex, and a minority of recorded cells was located in the ventral prelimbic cortex.

\section{DISCUSSION}

Here, we show that early post-weaning social experience is critical for the development of cognitive capacities and PFC function in rats. Deprivation of social contact during a period in which marked changes in social behavior take place (PND 21-42), resulted in disrupted impulse control in the 5-CSRTT and impaired decision making in the rGT in adulthood, even after a prolonged period of re-socialization. ISO rats were less sensitive to amphetamine and GBR12909 under demanding conditions in the 5-CSRTT. In line with the reduced sensitivity to enhanced dopaminergic neurotransmission, whole-cell recordings in slices from adult animals showed that early social isolation caused mPFC pyramidal neurons to become insensitive to modulation of synaptic response amplitude by dopamine.

Early social life events have profound repercussions for the development of brain and behavior, which increase the vulnerability for neuropsychiatric disorders in adulthood (Cacioppo and Hawkley, 2009; Paus et al, 2008). However, only a limited number of studies have investigated the importance of social behavior during the juvenile and early adolescent stages, which is a period characterized by substantial changes in the structure of social behavior (Blakemore, 2008; Nelson et al, 2005; Spear, 2000). In particular, this period of life is characterized by the abundance of social play behavior, which is thought to 


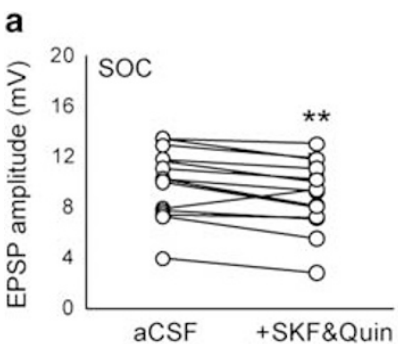

C

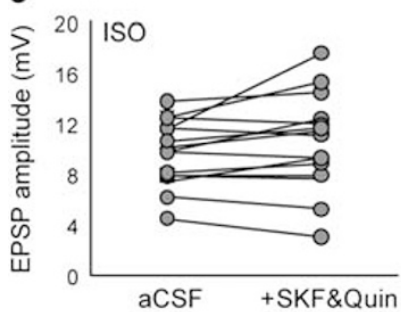

b
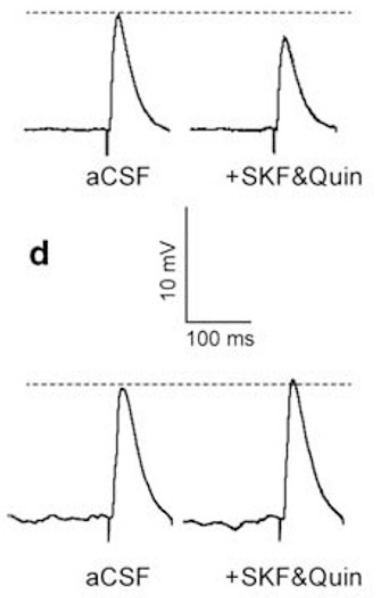
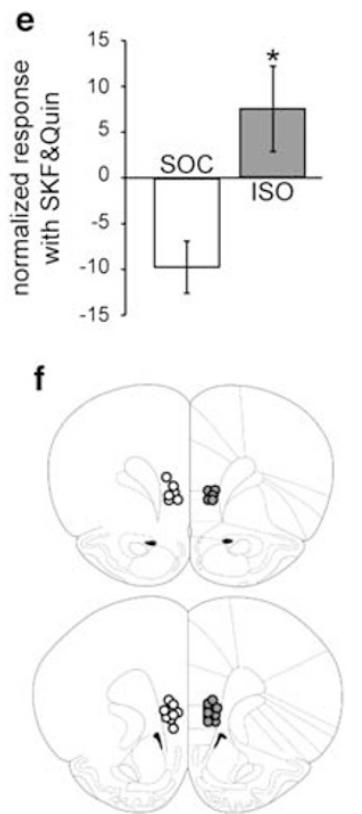

Figure 5 Effect of social isolation during postnatal day $21-42$ followed by re-socialization on synaptic response size in mPFC. EPSP amplitude before and after bath-application of a combination of SK38393 (5 $\mu$ M) and quinpirole (I $\mu$ M) in adult SOC animals $(a ; n=14$ cells from nine animals, double asterisks indicate $P<0.01$ compared with aCSF; b: example trace). EPSP amplitude in animals socially isolated between P2I and 42 (ISO; c; $n=17$ cells from eight animals; d: example trace). A summary of the EPSP amplitude following application of SKF38393 and quinpirole is shown in e. Location of pyramidal cells, mostly in infralimbic mPFC layer $V$, recorded from is shown in $f$ (white circles are cells from SOC animals, gray circles are cells from adult animals socially isolated from PND 2I-42; ISO). Asterisk indicates $P<0.05$ compared with SOC group. Data are expressed as mean \pm SEM.

contribute to the development of social and cognitive functions, and in particular to acquire the ability to flexibly use these capacities under changeable circumstances (Pellis and Pellis, 2009; Śpinka et al, 2001; Vanderschuren et al, 1997; Trezza et al, 2010). Previous work has shown that deprivation of social contact in post-weaning rats results in behavioral deficits within the social domain (Lukkes et al, 2009; Van den Berg et al, 1999). These changes lasted into adulthood, even when the isolation period was followed by re-socialization. The present observations extend these findings, as social isolation during the juvenile and early adolescent stages resulted in cognitive control deficits in adulthood. Impulsive action, measured as premature responses in the 5-CSRTT, was enhanced in ISO rats when test conditions were unexpectedly made more demanding (ie, long ITI and short stimulus duration). Moreover, early social isolation resulted in impaired decision making in a rodent variant of the Iowa gambling task. Both humans and SOC rats develop a preference for the advantageous options within the first session of the IGT or rGT, respectively (Baarendse et al, 2013; Bechara et al, 1994), but this acquisition curve was absent in ISO rats. Importantly, these impairments in cognitive control arose mainly under challenging (long ITI and short stimulus duration in the 5-CSRTT) or novel (first session in the rGT) circumstances. This is consistent with the hypothesis that the expression of social play behavior contributes to the ability to act flexibly in a changeable, dynamic environment (Špinka et al, 2001).

Previously, we showed that during long ITI conditions in the 5-CSRTT, amphetamine and the DA reuptake blocker GBR12909 enhanced, and the NA reuptake blocker atomoxetine and 5-HT reuptake blocker citalopram reduced impulsive action (Baarendse and Vanderschuren, 2012). Here, we found that early social isolation resulted in impaired impulse control and a reduced sensitivity to amphetamine and GBR12909, but not atomoxetine or citalopram, in the 5-CSRTT under long ITI conditions. These effects were selective for impulsive action, as neither attentional nor motivational processes in the 5-CSRTT, nor impulsive choice in the DRT were affected by early adolescent social isolation. In addition, the sensitivity to amphetamine and GBR12909 was not changed under baseline conditions in the 5-CSRTT, which underscores the notion that the consequences of early social isolation become especially apparent under challenging conditions. This suggests that dopamine function required for coping with sudden changes in task requirements (perhaps at the level of the mPFC) is compromised as a result of social isolation. The blunted response to amphetamine in the 5CSRTT is reminiscent of earlier findings in isolation-reared rats (Dalley et al, 2002a). However, unlike the present data, this latter study found no behavioral differences in the 5CSRTT under baseline and challenging conditions between isolation-reared and control rats. Together, these findings therefore suggest that post-weaning social isolation can induce long-lasting changes in impulse control, but that the exact pattern of behavioral changes depends on the precise period of social isolation. Thus, social isolation during a period in life when social play behavior is highly abundant causes persistent alterations in the neural circuits underlying the control of impulsive actions, in particular its dopaminergic mechanisms (Eagle and Baunez, 2010; Pattij and Vanderschuren, 2008).

Although several studies have shown altered dopaminergic function in the PFC and striatum by chronic social 
isolation (Fone and Porkess, 2008), evidence about the contribution of social behavior during the specific time period when social play behavior is highly abundant is scarce. Here, we showed that the behavioral deficits induced by social isolation during PND 21-42 were accompanied by a loss of sensitivity to dopamine in the mPFC. Thus, a combination of dopamine D1 and D2 receptor agonists reduced EPSP amplitude in mPFC pyramidal neurons from SOC animals, but not from ISO rats. Dopamine is a critical modulator of the efficacy of both excitatory and inhibitory synaptic activity in the PFC (Seamans and Yang, 2004). Fibers from dopamine neurons originating in the ventral tegmental area increasingly innervate the PFC through adolescence (Kalsbeek et al, 1988), and the modulation of PFC circuits by dopamine D1 and D2 receptors responsible for the excitation-inhibition balance changes considerably during post-weaning development (Brenhouse et al, 2008; Tseng and O'Donnell, 2007). The changes in dopamine modulation of synaptic responses that we observed following early social isolation are reminiscent of the adolescent phenotype where D2 receptor stimulation has no effect on excitatory synaptic transmission, which is dependent on the maturation of D2 receptors on interneurons (Tseng and O'Donnell, 2007). As activity-dependent processes of receptor and synaptic pruning during development finetune neural circuitry in an input-dependent manner (Lichtman and Colman, 2000), this suggests that the lack of social contact during early post-weaning development interferes with PFC maturation (see also Leussis et al, 2008; Makinodan et al, 2012). Whether the changed sensitivity for dopamine in the MPFC in the present study is dependent on the maturation of interneurons and causally related to the observed deficits in impulse control and decision making remains to be established. As yet, there is only scarce information about the role of dopamine in the PFC in the regulation of impulsive action and decision making in the 5CSRTT and rGT, respectively (Dalley et al, 2002b; Economidou et al, 2012). Interestingly, nucleus accumbens dopamine release has been shown to be potentiated when mPFC dopamine activity is reduced (Louilot et al, 1989; Mitchell and Gratton, 1992), suggesting an inverse relationship between dopamine activity in the MPFC and nucleus accumbens. Therefore, it could be that the behavioral deficits by early social isolation are due to altered dopamine activity in the nucleus accumbens as a result of disrupted PFC input into the mesoaccumbens dopamine system, as dopaminergic activity in the nucleus accumbens has been implicated in impulsive action in the 5-CSRTT (Cole and Robbins, 1989; Pattij et al, 2007).

Several limitations of the present study should be acknowledged. First, different strains of rats were used in the behavioral (Lister Hooded) and electrophysiological (Long Evans) experiments. Although it is reasonable to assume that the behavioral and electrophysiological changes induced by early social isolation are related (see above), future studies should pertinently address this issue, within a single strain of rats. Second, because of logistic reasons, the animals were socially isolated the day after arrival in the laboratory. Shipping stress may therefore have interfered with the effects of early social isolation. Interestingly, a recent study has shown that corticosterone treatment during PND 30-50 has long-lasting effects on impulsive behavior in the 5-CSRTT and DRT (Torregrossa et al, 2012), but the pattern of effects was different from that seen in the present study. This suggests that the effects of social isolation in the present study are not merely the result of stress during the juvenile and early adolescent stages, although further work is necessary to investigate this in more detail.

In conclusion, the present findings indicate that the lack of proper social experience during the juvenile and early adolescent stages of life has long-lasting effects on the function of PFC circuits underlying cognitive control. This impaired cognitive control may account for an increased vulnerability for psychiatric disorders as a result of early social insults (Cacioppo and Hawkley, 2009; Paus et al, 2008). Indeed, impulsivity and decision-making deficits have been associated with a variety of psychiatric disorders, including attention-deficit/hyperactivity disorder and drug addiction (Chamberlain and Sahakian, 2007; Moeller et al, 2001). Our results underscore that experimental approaches which investigate the link between social experiences and neurobehavioral outcomes can extend our understanding of the pathways leading to increased risk or resilience to psychiatric illness.

\section{ACKNOWLEDGEMENTS}

This study was supported by National Institute on Drug Abuse Grants R01 DA022628 (L.J.M.J.V.) and R01 DA014020 (P.O’D).

\section{DISCLOSURE}

The authors declare no conflict of intertest.

\section{REFERENCES}

Andersen SL (2003). Trajectories of brain development: point of vulnerability or window of opportunity? Neurosci Biobehav Rev 27: 3-18.

Baarendse PJJ, Vanderschuren LJMJ (2012). Dissociable effects of monoamine reuptake inhibitors on distinct forms of impulsive behavior in rats. Psychopharmacology 219: 313-326.

Baarendse PJJ, Winstanley CA, Vanderschuren LJMJ (2013). Simultaneous blockade of dopamine and noradrenaline reuptake promotes disadvantageous decision making in a rat gambling task. Psychopharmacology 225: 719-731.

Bechara A, Damasio AR, Damasio H, Anderson SW (1994). Insensitivity to future consequences following damage to human prefrontal cortex. Cognition 50: 7-15.

Blakemore SJ (2008). The social brain in adolescence. Nat Rev Neurosci 9: 267-277.

Brenhouse HC, Sonntag KC, Andersen SL (2008). Transient D1 dopamine receptor expression on prefrontal cortex projection neurons: relationship to enhanced motivational salience of drug cues in adolescence. J Neurosci 28: 2375-2382.

Cacioppo JT, Hawkley LC (2009). Perceived social isolation and cognition. Trends Cogn Sci 13: 447-454.

Casey BJ, Tottenham N, Liston C, Durston S (2005). Imaging the developing brain: what have we learned about cognitive development? Trends Cogn Sci 9: 104-110.

Chamberlain SR, Sahakian BJ (2007). The neuropsychiatry of impulsivity. Curr Opin Psychiatry 20: 255-261.

Cole BJ, Robbins TW (1989). Effects of 6-hydroxydopamine lesions of the nucleus accumbens septi on performance of a 5-choice 
serial reaction time task in rats: implications for theories of selective attention and arousal. Behav Brain Res 33: 165-179.

Counotte DS, Li KW, Wortel J, Gouwenberg Y, Van Der Schors RC, Smit $\mathrm{AB}$ et al (2010). Changes in molecular composition of rat medial prefrontal cortex synapses during adolescent development. Eur J Neurosci 32: 1452-1460.

Dalley JW, Theobald DE, Eagle DM, Passetti F, Robbins TW (2002b). Deficits in impulse control associated with tonicallyelevated serotonergic function in rat prefrontal cortex. Neuropsychopharmacology 26: 716-728.

Dalley JW, Theobald DE, Pereira EA, Li PM, Robbins TW (2002a). Specific abnormalities in serotonin release in the prefrontal cortex of isolation-reared rats measured during behavioural performance of a task assessing visuospatial attention and impulsivity. Psychopharmacology 164: 329-340.

Eagle DM, Baunez C (2010). Is there an inhibitory-responsecontrol system in the rat? Evidence from anatomical and pharmacological studies of behavioral inhibition. Neurosci Biobehav Rev 34: 50-72.

Economidou D, Theobald DE, Robbins TW, Everitt BJ, Dalley JW (2012). Norepinephrine and dopamine modulate impulsivity on the five-choice serial reaction time task through opponent actions in the shell and core sub-regions of the nucleus accumbens. Neuropsychopharmacology 37: 2057-2066.

Evenden JL, Ryan CN (1996). The pharmacology of impulsive behaviour in rats: the effects of drugs on response choice with varying delays of reinforcement. Psychopharmacology 128: 161-170.

Fone KC, Porkess MV (2008). Behavioural and neurochemical effects of post-weaning social isolation in rodents-relevance to developmental neuropsychiatric disorders. Neurosci Biobehav Rev 32: 1087-1102.

Kalsbeek A, Voorn P, Buijs RM, Pool CW, Uylings HB (1988). Development of the dopaminergic innervation in the prefrontal cortex of the rat. J Comp Neurol 269: 58-72.

Leussis MP, Lawson K, Stone K, Andersen SL (2008). The enduring effects of an adolescent social stressor on synaptic density, part II: Poststress reversal of synaptic loss in the cortex by adinazolam and MK-801. Synapse 62: 185-192.

Lichtman JW, Colman H (2000). Synapse elimination and indelible memory. Neuron 25: 269-278.

Louilot A, Le Moal M, Simon H (1989). Opposite influences of dopaminergic pathways to the prefrontal cortex or the septum on the dopaminergic transmission in the nucleus accumbens. An in vivo voltammetric study. Neuroscience 29: 45-56.

Lukkes J, Vuong S, Scholl J, Oliver H, Forster G (2009). Corticotropin-releasing factor receptor antagonism within the dorsal raphe nucleus reduces social anxiety-like behavior after early-life social isolation. J Neurosci 29: 9955-9960.

Makinodan M, Rosen KM, Ito S, Corfas G (2012). A critical period for social experience-dependent oligodendrocyte maturation and myelination. Science 337: 1357-1360.

McCutcheon JE, Marinelli M (2009). Age matters. Eur J Neurosci 29: 997-1014.

Miller EK, Cohen JD (2001). An integrative theory of prefrontal cortex function. Annu Rev Neurosci 24: 167-202.

Mitchell JB, Gratton A (1992). Partial dopamine depletion of the prefrontal cortex leads to enhanced mesolimbic dopamine release elicited by repeated exposure to naturally reinforcing stimuli. J Neurosci 12: 3609-3618.

Moeller FG, Barratt ES, Dougherty DM, Schmitz JM, Swann AC (2001). Psychiatric aspects of impulsivity. Am J Psychiatry 158: 1783-1793.

Nelson EE, Leibenluft E, McClure EB, Pine DS (2005). The social re-orientation of adolescence: a neuroscience perspective on the process and its relation to psychopathology. Psychol Med 35: 163-174.

Panksepp J (1981). The ontogeny of play in rats. Dev Psychobiol 14: 327-332.

Pattij T, Janssen MCW, Vanderschuren LJMJ, Schoffelmeer ANM, van Gaalen MM (2007). Involvement of dopamine D1 and D2 receptors in the nucleus accumbens core and shell in inhibitory response control. Psychopharmacology 191: 587-598.

Pattij T, Vanderschuren LJMJ (2008). The neuropharmacology of impulsive behaviour. Trends Pharmacol Sci 29: 192-199.

Paus T, Keshavan M, Giedd JN (2008). Why do many psychiatric disorders emerge during adolescence? Nat Rev Neurosci 9: 947-957.

Pellis SM, Pellis V (2009). The playful brain: venturing to the limits of neuroscience. Oneworld Publications: Oxford, UK.

Robbins TW (2002). The 5-choice serial reaction time task: behavioural pharmacology and functional neurochemistry. Psychopharmacology 163: 362-380.

Robbins TW, Arnsten AFT (2009). The neuropsychopharmacology of fronto-executive function: monoaminergic modulation. Annu Rev Neurosci 32: 267-287.

Seamans JK, Yang CR (2004). The principal features and mechanisms of dopamine modulation in the prefrontal cortex. Prog Neurobiol 74: 1-58.

Spear LP (2000). The adolescent brain and age-related behavioral manifestations. Neurosci Biobehav Rev 24: 417-463.

Špinka M, Newberry RC, Bekoff M (2001). Mammalian play: training for the unexpected. Q Rev Biol 76: 141-168.

Torregrossa MM, Xie M, Taylor JR (2012). Chronic corticosterone exposure during adolescence reduces impulsive action but increases impulsive choice and sensitivity to yohimbine in male Sprague-Dawley rats. Neuropsychopharmacology 37: 1656-1670.

Trezza V, Baarendse PJJ, Vanderschuren LJMJ (2010). The pleasures of play: pharmacological insights into social reward mechanisms. Trends Pharmacol Sci 31: 463-469.

Tseng KY, Lewis BL, Hashimoto T, Sesack SR, Kloc M, Lewis DA et al (2008). A neonatal ventral hippocampal lesion causes functional deficits in adult prefrontal cortical interneurons. J Neurosci 26: 12691-12699.

Tseng KY, O'Donnell P (2005). Post-pubertal emergence of prefrontal cortical oscillations induced by $\mathrm{D}_{1}$-NMDA co-activation. Cerebral Cortex 15: 49-57.

Tseng KY, O’Donnell P (2007). Dopamine modulation of prefrontal cortical interneurons changes during adolescence. Cerebral Cortex 17: 1235-1240.

Van den Berg CL, Hol T, Van Ree JM, Spruijt BM, Everts H, Koolhaas JM (1999). Play is indispensable for an adequate development of coping with social challenges in the rat. Dev Psychobiol 34: 129-138.

Van Gaalen MM, Brueggeman RJ, Bronius PFC, Schoffelmeer ANM, Vanderschuren LJMJ (2006a). Behavioral disinhibition requires dopamine receptor activation. Psychopharmacology 187: 73-85.

Van Gaalen MM, van Koten R, Schoffelmeer ANM, Vanderschuren LJMJ (2006b). Critical involvement of dopaminergic neurotransmission in impulsive decision making. Biol Psychiatry 60: 66-73.

Vanderschuren LJMJ, Niesink RJM, Van Ree JM (1997). The neurobiology of social play behavior in rats. Neurosci Biobehav Rev 21: 309-326.

Zeeb FD, Robbins TW, Winstanley CA (2009). Serotonergic and dopaminergic modulation of gambling behavior as assessed using a novel rat gambling task. Neuropsychopharmacology 34: 2329-2343. 